\title{
Influence of Capillary Number on the droplet Shape, Film Thickness, and Pressure Drop in a Liquid-Liquid Taylor Flow inside a Microcapillary
}

\author{
S. V. B. Vivekanand \\ Mechanical Engineering Department, \\ National Institute of Technology, Warangal, \\ Telangana State - 506004, India \\ S. Chandrasekhar \\ Mechanical Engineering Department, \\ National Institute of Technology, Warangal, \\ Telangana State - 506004, India \\ V. R. K. Raju \\ Mechanical Engineering Department, \\ National Institute of Technology, Warangal, \\ Telangana State - 506004, India \\ Corresponding author: vrkraju9575@gmail.com
}

(Received December 24, 2018; Accepted August 9, 2019)

\begin{abstract}
Numerical analysis of a two-dimensional, axisymmetric, incompressible, laminar liquid-liquid Taylor flow inside a vertical circular microchannel is carried out in the present study. The focus is laid on fluid flow characteristics in the slug flow regime. Although many researchers have performed numerical and experimental studies of two-phase flows in narrow channels, their efforts seem to have been fairly successful in explaining the underlying mechanisms of fluid flow phenomena, especially for slug flow regime. Here, an attempt has been made to explore the hydrodynamics of such flows. In the present study, dodecane and Pd5 have been used as the carrier phases and water is used as the discontinuous phase. The internal diameter of the circular microchannel is $1.5 \mathrm{~mm}$ with its wall being insulated. The flow and volume fraction equations are solved by the finite volume approach (FVM). The volume of fluid (VOF) method has been adopted for capturing the interface. The effect of Capillary number on film thickness and interfacial pressure drop is explained. The film thickness is found to increase with Capillary number and is also found to be in a close match with the models available in the literature. The pressure drop per unit length obtained from the CFD study is compared with a standard model available in the literature. The pressure drop across the unit cell is found to be following the phenomenological model. It is observed that the pressure drop at the interface has the highest contribution to the total pressure drop in contrast to the other pressure drops in the channel, with $~ 50-55 \%$ in dodecanewater and $~ 55-62 \%$ in Pd5-water systems. Besides, the distribution of the velocity, axial, and wall pressure fields inside the microcapillary are also discussed.
\end{abstract}

Keywords- Film thickness, Pressure drop, Taylor flow, Volume of fluid method.

\section{Introduction}

Two-phase slug flow, whether it is liquid-liquid or gas-liquid, is used for the removal of high heat fluxes at the expense of low coolant flow rate. The potentiality of these flows with respect to the liquid-only flows has been studied and reported by many researchers (Triplett et al., 1999; Asadolahi et al., 2011; Asadolahi et al., 2012). Taylor flow (Slug flow) regime is considered to be an important flow regime since it covers a significant portion in a flow regime map (Triplett et 
International Journal of Mathematical, Engineering and Management Sciences

Vol. 4, No. 6, 1407-1419, 2019

https://dx.doi.org/10.33889/IJMEMS.2019.4.6-111

al., 1999). Taylor flow is characterized by a sequence of liquid droplets (or bubbles) filling the entire flow channel with liquid slugs between two consecutive droplets. In Taylor flows, the presence of a thin liquid film between the wall and droplets, and Taylor vortices inside the liquid slugs are the key factors for the augmentation in the heat and mass transfer rates. In gas-liquid flows, the role of the gas phase in the heat transfer process is often neglected due to its lower thermal conductivity. Introducing an immiscible liquid droplet in place of gas bubble significantly increases the heat transfer rates. Asthana et al. (2011), Ookawara et al. (2007), Song et al. (2006), and Urbant et al. (2008) have discussed a wide variety of applications of liquidliquid slug flows, such as in polymerase chain reaction, Nitration of benzene to toluene, micro separation, and electronics cooling, respectively.

A wide number of numerical and experimental studies have been performed by eminent researchers to explore the hydrodynamics of gas-liquid as well as liquid-liquid Taylor flows. In experimental studies, the recirculation within the liquid slugs can be seen by Particle Image Velocimetry (PIV) technique (Kashid et al., 2005), whereas in numerical studies these can be seen in droplet/bubble frame of reference (Asadolahi et al., 2011). Taha and Cui (2006) implemented VOF technique to describe the motion of gas bubbles in stagnant as well as flowing liquids and validated their results with the published experimental results of White and Beardmore (1962). Gupta et al. (2009) performed simulations on a two-dimensional, horizontal, axisymmetric domain $(\mathrm{d}=0.5 \mathrm{~mm}, \mathrm{~L}=10 \mathrm{~d})$ using VOF multiphase method to explore the hydrodynamics of air-water Taylor flows inside the microchannel. They developed a methodology for simulating slug flows using the VOF method. In their numerical work on slug flows in T-Junction microchannels, Santos and Kawaji (2010); Nichita et al. (2010) numerically studied the effect of wall wettability on the flow physics of gas-liquid Taylor flows in a Tjunction microchannel. Farhangi et al. (2010) studied numerically the hydrodynamics of a single bubble rising in a vertical tube using a two-dimensional axisymmetric model. They investigated the effect of different parameters such as surface tension, channel height, initial bubble radius and liquid viscosity on the shape and velocity of the bubble. Through their micro-PIV experiments, and numerical studies on gas-liquid Taylor flows in a horizontal circular channel, Gupta et al. (2013b) explained the significance of the third dimension on the bubble shape and fluid flow. An asymmetry was observed in the bubble shape which was found to be in close agreement with their experimental results.

Based on this literature survey it is understood that most of the studies are done on gas-liquid slug flows, whereas only a few studies have been conducted with liquid-liquid flows. Liquid-liquid flows are efficient in dissipating high heat fluxes and also promote the mixing performance due to the higher thermal conductivity of dispersed liquid unlike the gas phase in gas-liquid flows. This study is aimed at a systematic simulation of liquid-liquid Taylor flows in a circular microchannel to acquire a thorough knowledge of the flow characteristics in microchannels. The study numerically investigates the dependence of the two-phase flow on important flow parameters such as homogeneous void fraction, slug and droplet lengths, and Capillary number. The corresponding numerical results have also been compared with widely accepted correlations.

\section{Simulation Methods}

\subsection{Governing Equations}

The present work deals with a two-dimensional, axisymmetric (x-r) liquid-liquid slug flow (or droplet-train flow) inside a circular microchannel where both phases are liquids. Each of the two fluids is considered to be incompressible, Newtonian, immiscible and without undergoing any 
International Journal of Mathematical, Engineering and Management Sciences

Vol. 4, No. 6, 1407-1419, 2019

https://dx.doi.org/10.33889/IJMEMS.2019.4.6-111

phase change. Volume-of-fluids (VOF) interface capturing technique (Hirt and Nichols, 1981) has been adopted to track the two-phase interface between the fluids. The model has been extensively used by several groups of researchers (Gupta et al., 2009; Gupta et al., 2013a; Vivekanand and Raju, 2015). The governing equations of the flow and volume fraction, as well as the surface tension modeling (Brackbill et al., 1992) which are solved by the solver, can be obtained from Gupta et al. (2009).

\subsection{Computational Domain}

Fully-Developed liquid-liquid slug flow can be modelled in a periodic unit cell due to its periodic and regular nature (Asadolahi et al., 2011). The periodic unit cell consists of two halves of continuous phase liquid at either end of the disperse phase droplet which is situated at the centre of the unit cell. The position of dispersed phase droplet remains unchanged in the unit cell when observed from a reference frame moving with a velocity equal to that of the droplet. Hence, the flow can be considered as steady in the moving frame of reference. Figure 1 shows the abovementioned unit cell having a periodic inlet and outlet at its ends and Figure 2 shows the cut plot of the fully developed unit cell obtained in the CFD study.

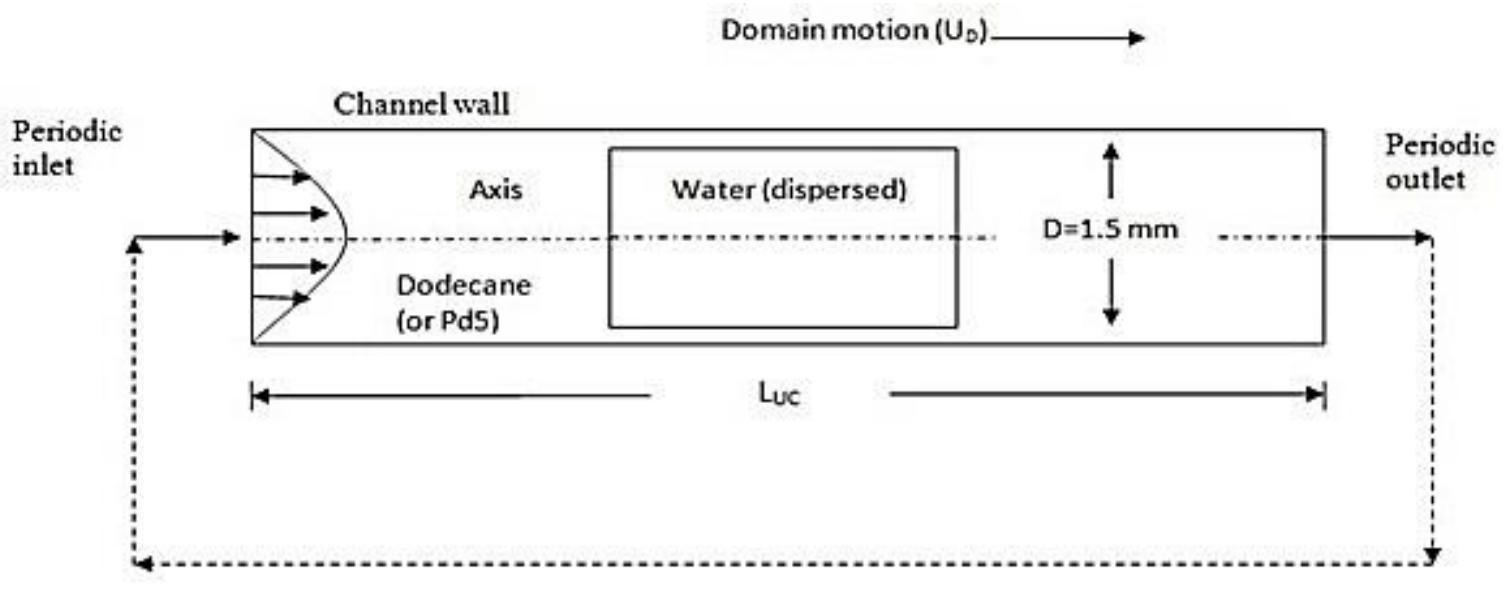

Figure 1. Schematic diagram of the periodic unit cell

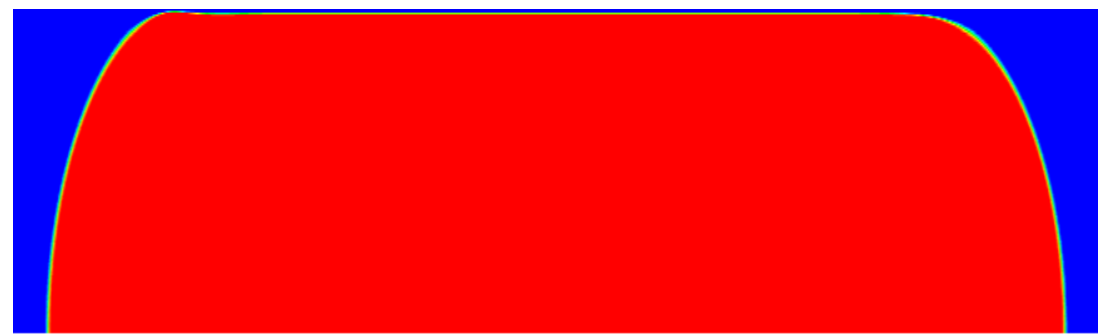

Figure 2. Cut-plot of CFD model. The red-coloured region shows the dispersed phase droplet, and the bluecoloured region shows primary phase fluid 
International Journal of Mathematical, Engineering and Management Sciences

Vol. 4, No. 6, 1407-1419, 2019

https://dx.doi.org/10.33889/IJMEMS.2019.4.6-111

\subsection{Methodology}

\subsubsection{Moving Domain Method}

The dynamic mesh motion method, available in ANSYS Fluent, is incorporated to model the twophase flow due to its lower computational cost over stationary domain method. The moving domain method is employed since the flow domain moves with the droplet velocity $\left(U_{\mathrm{D}}\right)$, and hence, the shape of the domain also changes over time. Rigid body treatment is used as both the domain as well as the incoming stream move in the same direction. Besides, the dynamic layering technique is adapted to update the mesh at each time step. It should be noted that at the beginning of each time-step, the droplet velocity is calculated using Eq. (1) (Asadolahi et al., 2011) and is supplied to the solver by user-defined $C$ routines. The homogeneous void fraction $(\beta)$ can be obtained by Eq. (2) (Asadolahi et al., 2011).

$$
\begin{aligned}
& U_{D}=\frac{\int_{x}^{x+L_{U C}} \int_{0}^{R} \alpha_{D} u_{x}(2 \pi r) d r d x}{\int_{x}^{x+L_{U C}} \int_{0}^{R} \alpha_{D}(2 \pi r) d r d x} \\
& \alpha_{D} U_{D}=\beta u_{T P}
\end{aligned}
$$

where, $\alpha_{\mathrm{D}}$ is the volume fraction of the disperse phase droplet, and $\mathbf{u}_{\mathrm{TP}}$ is the mixture velocity in $\mathrm{m} / \mathrm{s}$.

\subsubsection{Boundary and Initial Conditions}

Periodic velocity inlet and pressure outlet boundary conditions are applied at both ends with noslip at the channel wall. The radial and axial velocity components are transformed into the upstream boundary from the downstream boundary. The continuous phase liquid is allowed to enter the domain at a constant velocity. At the beginning of each simulation, the solution domain has a liquid droplet having a size equal to the required volume fraction. The entire flow channel is initialized with a uniform pressure of zero Pascal and fully-developed parabolic velocity profile having an average mixture velocity equal to the incoming primary (continuous) phase fluid. The periodic velocity boundary condition mentioned above is achieved by wrapping the axial and radial velocity fields from the downstream boundary to the upstream boundary using user-defined $\mathrm{C}$ routines in the solver as shown in the equations below:

$u_{x, r}=u_{x+L_{U C}, r}$

$v_{x, r}=v_{x+L_{U C}, r}$

\subsubsection{Numerical Schemes}

The commercial code ANSYS Fluent 15.0 was employed to perform this study. The volume of fluid (VOF) multiphase method was adopted to capture the interface between the two phases. An implicit scheme of the first-order with non-iterative solver was used for the unsteady formulation of flow equations. Green-Gauss node-based method was used to resolve the gradients of flow parameters. The interface reconstruction was achieved by using Geo-Reconstruct algorithm. A maximum Courant number of 0.25 was adapted for the volume fraction equation. Variable timestepping strategy with global Courant number of 0.25 was used for momentum, pressure and energy equations. Body force weighted scheme for solving pressure equation, and QUICK scheme for solving the convective terms of momentum equations was employed. The surface 
International Journal of Mathematical, Engineering and Management Sciences

Vol. 4, No. 6, 1407-1419, 2019

https://dx.doi.org/10.33889/IJMEMS.2019.4.6-111

tension coefficients at the Do-W and Pd5-water interfaces were 0.052 and $0.0397 \mathrm{~N} / \mathrm{m}$, respectively.

\section{Results and Discussion}

In the present work, dodecane and Pd5 are considered as the continuous phase liquids and water as the dispersed phase liquid. The thermophysical properties of the working fluids are listed in Table 1 . The diameter of the circular channel is $1.5 \mathrm{~mm}$. The simulation is performed for different dispersed phase volume fraction $0.15<\alpha_{\mathrm{D}}<0.75$, and mixture velocities $0.0566<\mathrm{u}_{\mathrm{TP}}<0.113$ (for dodecane-water system) and $0.0168<\mathrm{u}_{\mathrm{TP}}<0.0335$ (for Pd5-water system), whereas, the Capillary number in both cases of working fluids is identical and lies in the range $0.0015<\mathrm{Ca}_{\mathrm{C}}<0.00302$. A single rectangular droplet is initially placed in the computational domain. Each simulation begins with a continuous liquid (dodecane or Pd5) and dispersed droplet at rest.

Table 1. Thermophysical properties of the fluids used in the present study

\begin{tabular}{|l|c|c|c|}
\hline Material & $\begin{array}{l}\text { Density } \\
{\left[\mathrm{kg} / \mathrm{m}^{3}\right]}\end{array}$ & $\begin{array}{c}\text { Viscosity } \\
{[\mathrm{kg} / \mathrm{ms}]}\end{array}$ & $\begin{array}{c}\text { Interfacial Tension } \\
{[\mathrm{N} / \mathrm{m}]}\end{array}$ \\
\hline Water & 996.1 & 0.00091 & ---- \\
\hline Dodecane & 754.3 & 0.00139 & 0.0520 \\
\hline Pd5 & 911.8 & 0.00358 & 0.0397 \\
\hline
\end{tabular}

\subsection{Computational Mesh}

The computational mesh (Figure 3) used in the simulations consists of 85,000 square elements. Near-wall mesh treatment is performed by following the guidelines of Gupta et al. (2009) to capture the thin liquid film between the wall and droplet. Figure 4 shows the droplet shape for three different mesh sizes, viz. 65000, 85000, and 100,000 elements. It can be seen that the droplet shape obtained from 85000 and 100000 elements size are the same and the mesh having 85,000 elements has been adopted throughout the study.

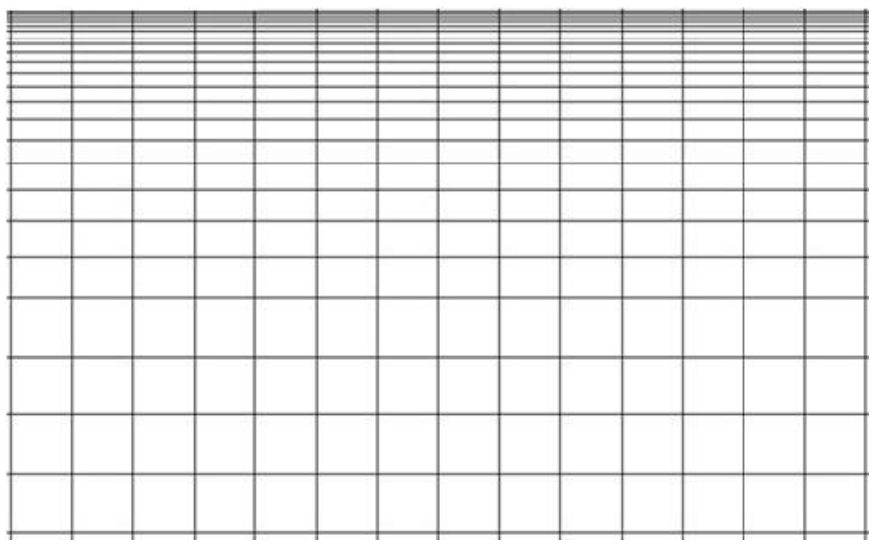

Figure 3. Computational mesh 
International Journal of Mathematical, Engineering and Management Sciences

Vol. 4, No. 6, 1407-1419, 2019

https://dx.doi.org/10.33889/IJMEMS.2019.4.6-111

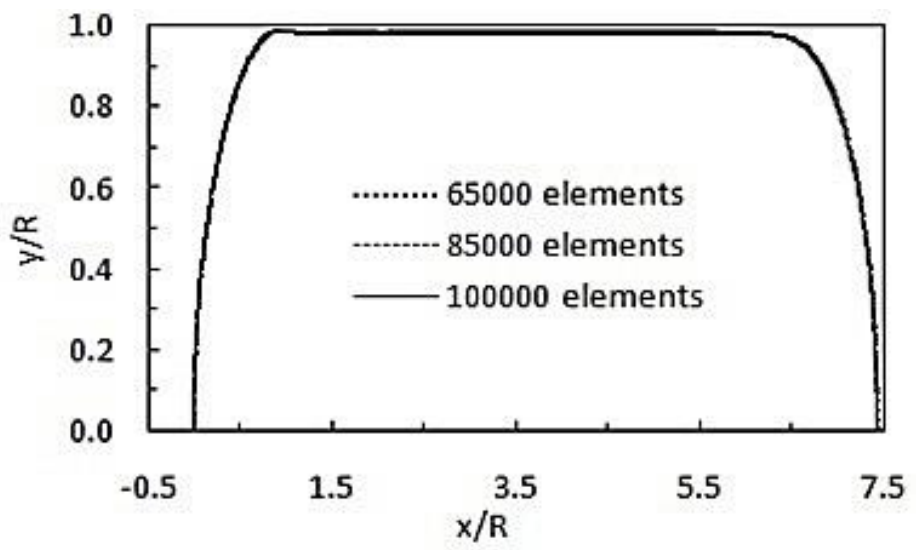

Figure 4. Droplet shape for different mesh sizes

\subsection{Film Thickness}

The CFD results of film thickness neighbouring a liquid droplet have been compared with Bretherton's free slip model (Bretherton, 1961) and Aussillous and Quere's (A\&Q) semiempirical correlation (Aussillous and Quere, 2000). Bretherton developed the model to evaluate the film thickness with an assumption of $\delta_{\mathrm{F}} \ll \mathrm{R}$, whereas, A\&Q proposed their model by relaxing the Bretherton's hypothesis. Variation of film thickness with the Capillary number (for Do-W case) is depicted in Figure 5. It is observed that film thicknesses near the water droplet, obtained from CFD simulations, are in accordance with Bretherton's free slip model of gas-liquid flow. Although Bretherton's model is applicable for gas-liquid Taylor flows, present simulation results were found to be in a close match with the model, having a mean absolute deviation of less than 4.70 percent, because of the lower viscosity ratio in the present case (Gupta et al., 2013a). Throughout the simulations, the homogeneous void fraction was kept constant $(\beta=0.753)$ and the average mixture velocity at the inlet was varied in the range $0.057-0.1132 \mathrm{~m} / \mathrm{s}$. In addition, a region of non-uniform film thickness was observed for lower values of the homogeneous void fraction $(\beta=0.2)$ which is in accordance with the previous literature (Shah and London, 1978; Lac and Sherwood, 2009; Fouilland et al., 2010).

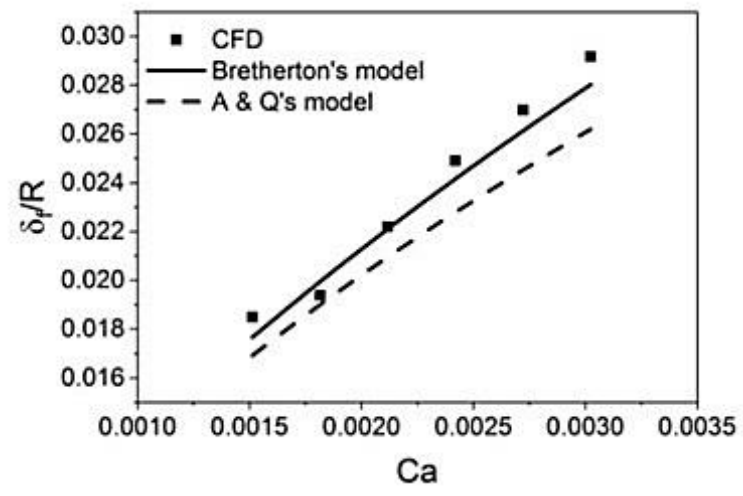

Figure 5. Variation of the continuous phase film thickness with the Capillary number (Do-W system) 
International Journal of Mathematical, Engineering and Management Sciences

Vol. 4, No. 6, 1407-1419, 2019

https://dx.doi.org/10.33889/IJMEMS.2019.4.6-111

\subsection{Droplet Shape}

The droplet profiles at different Capillary numbers $\left(0.057<\mathrm{u}_{\mathrm{TP}}<0.1132 \mathrm{~m} / \mathrm{s}\right)$ obtained from CFD simulations for Do-W system are shown in Figures 6 and 7. It is observed that the film thickness between the water droplet and channel wall increases with the Capillary number. The possible reason for this relationship is that the curvature of the leading meniscus (droplet nose) increases when the mixture velocity is increased, whereas, the curvature of the trailing meniscus (droplet tail) of the droplet is constant. For all the Capillary numbers considered in the study, a ripple (waves or undulations) at the rear end of the droplet was observed. These ripples indicate the zones of sharp pressure jumps near the interface. Similar observations were reported by Bretherton (1961) in their gas-liquid slug flow studies. Figures 6 and 7 also show the variation of wall pressure along the axial length of the channel at different mixture velocities for a given $\beta$. It can be seen that the pressure difference over the frontal region is higher than the pressure drop over the rear region.

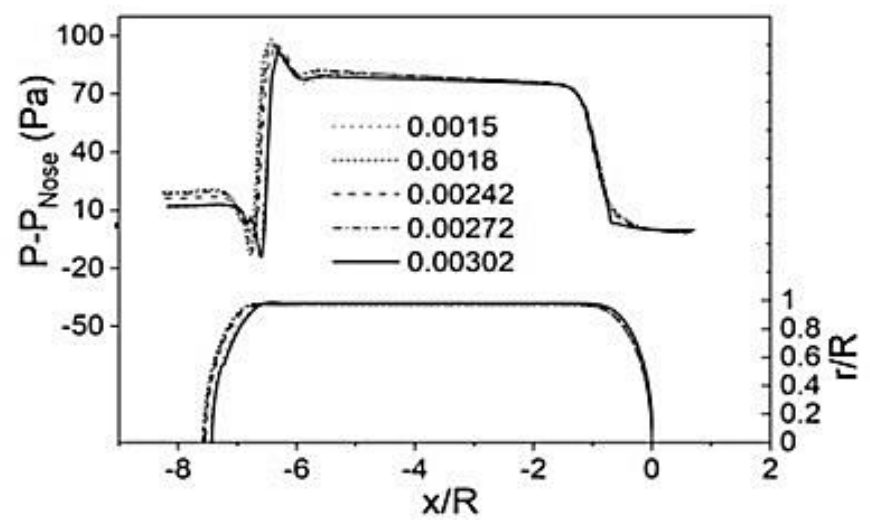

Figure 6. Variation of wall pressure and droplet shape along the axial length of the channel for different Capillary number at $\beta=0.735$ when the pressure at the nose of the droplets are transformed to zero and the nose of the droplets are made coincident

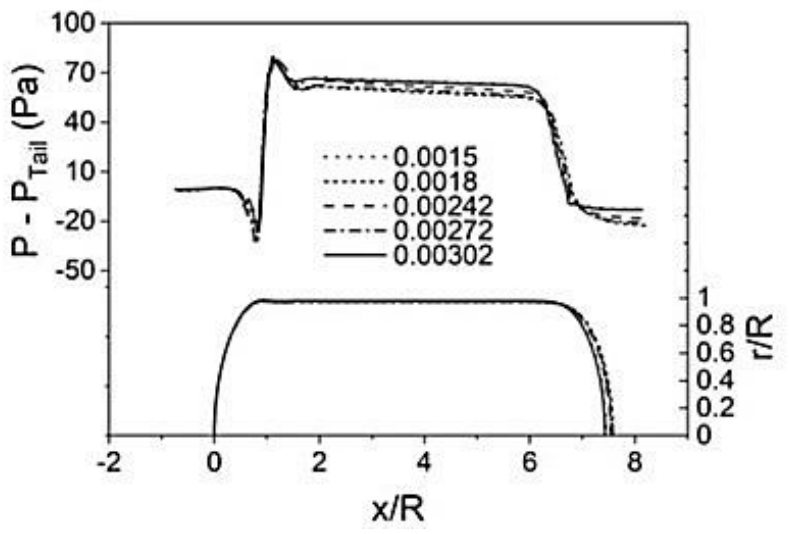

Figure 7. Variation of wall pressure and droplet shape along the axial length of the channel for different Capillary number at $\beta=0.735$ when the minimum pressure near the tail of the droplets is transformed to zero and the tails of the droplets are made coincident 
International Journal of Mathematical, Engineering and Management Sciences

Vol. 4, No. 6, 1407-1419, 2019

https://dx.doi.org/10.33889/IJMEMS.2019.4.6-111

\subsection{Pressure Field}

In this section, an effort has been made to study the pressure field in the two-phase liquid-liquid Taylor flow. The total pressure drop during the two-phase flow occurs due to three major contributing factors: (a) slug $\left(\Delta \mathrm{P}_{\text {slug }}\right)$; (b) film $\left(\Delta \mathrm{P}_{\text {film }}\right)$; and (c) the interfacial $\left(\Delta \mathrm{P}_{\text {interface }}\right)$ pressure drops (Gupta et al., 2013a). Slug pressure drop can be evaluated from Hagen-Poiseuille's equation (Eq. 5) due to fully developed flow in the primary phase slug. Figure 8 shows the variation of the wall and axial pressure along the axial length of the channel. It is observed that, in the region of uniform film thickness, the pressure gradients are identical in both the phases.

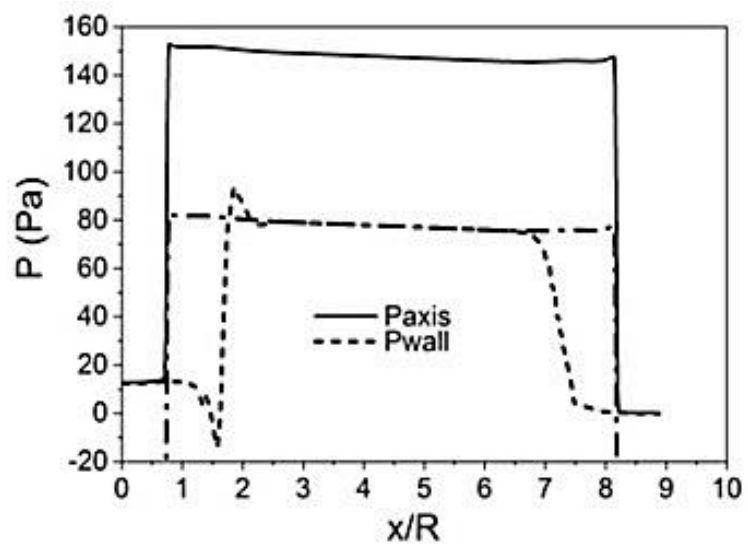

Figure 8. Variation of the wall and axial pressure along the axial length of the channel for $\mathbf{u}_{\mathrm{TP}}=0.0566 \mathrm{~ms}^{-1}$ and $\beta=0.735$ for the dodecane-water system. (The dashed line represents the axial pressure shifted by

Laplace pressure difference, which is equal to $75 \mathrm{~Pa}$ (approx.)

Fouilland et al. (2010) assumed random pressure gradients at the annulus and core, and have given a solution for velocity profile using an analytical, annular, laminar-laminar flow solution for gas-liquid Taylor flows, whereas, Gupta et al. (2013a) derived an expression for evaluating the film pressure drop (Eq. 6) considering equal pressure gradients unlike arbitrary gradients. The interfacial pressure drop could be calculated using Bretherton's model (Eq. 7).

$\Delta P_{\text {slug }}=\frac{8 \mu_{c} L_{s} u_{T P}}{R^{2}}$

$\Delta P_{f i l m}=\frac{8 \mu_{c} L_{f i l m} u_{T P}}{R^{2}\left(1+\left[\left(R_{i}{ }^{4}\right) /\left(R^{4}\right)\right][(1 / \lambda)-1]\right.}$

$\Delta P_{\text {interface }}=4.52 \frac{\sigma}{R}(3 C a)^{2 / 3}$

Figures 9 and 10 show the comparison of interfacial pressure drop across the interface between the two fluids obtained from simulations with that obtained from Eq. (7). The interfacial pressure drop could be calculated by differencing the pressure between the droplet nose and tail. In all the cases considered in the present work, interfacial pressure drop has a significant contribution $(\sim 50$ - 
International Journal of Mathematical, Engineering and Management Sciences

Vol. 4, No. 6, 1407-1419, 2019

https://dx.doi.org/10.33889/IJMEMS.2019.4.6-111

$55 \%$ and $\sim 55-62 \%$ for dodecane-water and Pd5-water cases, respectively) in the overall pressure drop in contrast to other pressure drops.

$$
\begin{gathered}
\left(\frac{\Delta P}{L}\right)_{U C}=\frac{8 \mu_{c} u_{T P}}{R^{2}} \frac{L_{s}}{L_{U C}}+\frac{8 \mu_{c} u_{T P}}{R^{2}\left[\left(1+\left(R_{i}{ }^{4}\right) /\left(R^{4}\right)\right)((1 / \lambda)-1)\right]} \frac{L_{f i l m}}{L_{U C}} \\
+\frac{4.52}{L_{U C}} \frac{\sigma}{R}(3 C a)^{2 / 3}
\end{gathered}
$$

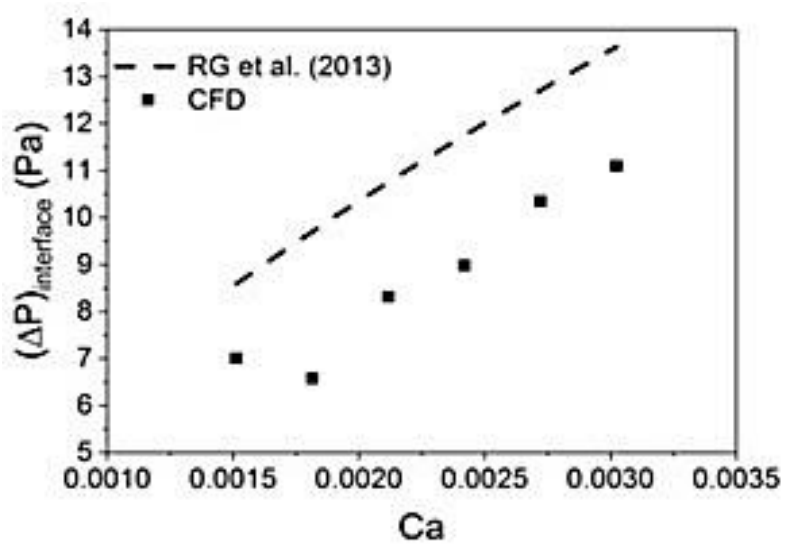

Figure 9. Comparison of the interfacial pressure drop as a function of Capillary number between the present CFD results and that obtained from Eq. (7) dodecane-water system

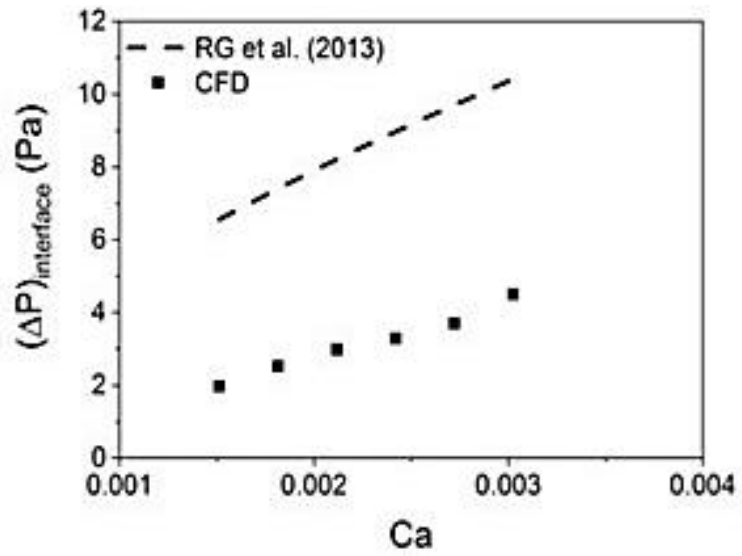

Figure 10. Comparison of the interfacial pressure drop as a function of Capillary number between the present CFD results and that obtained from Eq. (7) for Pd5-water system

Figure 11 compares the normalized pressure drop in the microchannel obtained from Eq. (8) and present simulations. In both simulations as well as the analytical model, it is observed that the normalized pressure drop $(\Delta \mathrm{P} / \mathrm{L})$ increases with mixture velocity. However, the analytical model 
International Journal of Mathematical, Engineering and Management Sciences

Vol. 4, No. 6, 1407-1419, 2019

https://dx.doi.org/10.33889/IJMEMS.2019.4.6-111

has a higher rate of increase as compared to CFD results. An approximate maximum absolute deviation of $20 \%$ and $40 \%$ between the CFD results of overall pressure drop and that obtained using the analytical model has been observed for Do-W and Pd5-W systems, respectively. Also, a significant deviation in the interfacial pressure drop is observed between the numerical results and those obtained from Bretherton's model for an inviscid bubble where the viscosity ratio for air-water system was 0.02 . The CFD results of overall pressure drop for hexadecane-water system (viscosity ratio 0.296) in the studies of Gupta et al. (2013a) were found be lying within $25 \%$ with respect to that evaluated from Eq. (8). However, in the present studies, these deviations are approximately $20 \%$ and $39 \%$, respectively for dodecane-water (viscosity ratio 0.655 ) and Pd5water (viscosity ratio 0.254 ) systems. These deviations emphasize the crucial role of viscosity ratio in the formulation of a pressure drop model. Hence, it is highly recommended to look for improvised models for predicting the interfacial and overall pressure drop for a liquid-liquid Taylor flow covering lower as well as a higher range of viscosity ratios.

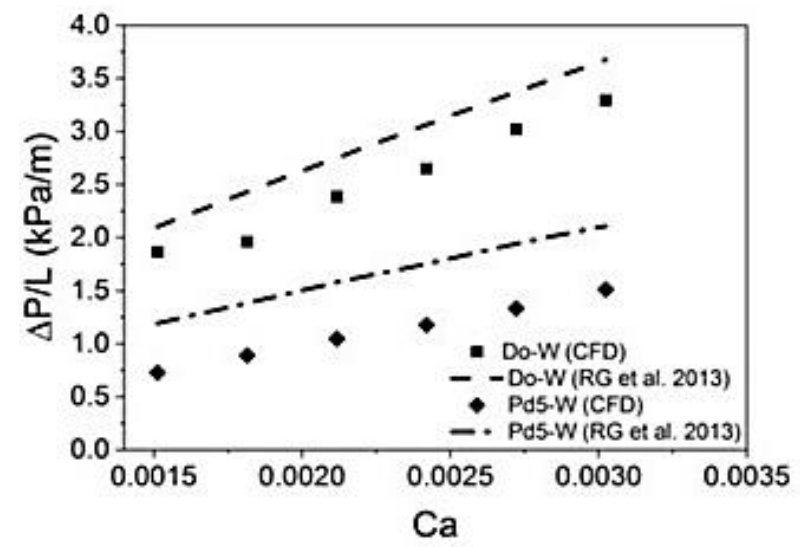

Figure 11. Comparison of pressure drop per unit length as a function of Capillary number between the present CFD results and that obtained from Eq. (8)

\subsection{Velocity Field}

Figure 12 shows the effect of mixture velocity on the velocity field along the radial direction in the middle of a slug. Besides, shown in the figure are the normalized velocity profiles of fullydeveloped laminar liquid-only flow and the droplet velocity $\left(\mathrm{U}_{\mathrm{D}} / \mathrm{u}_{\mathrm{TP}}\right)$ (horizontal dash-dotted line) for a mixture velocity of $0.0566 \mathrm{~m} / \mathrm{s}$. The velocity profiles indicate that the velocity at the axis has reached 1.8 times the average velocity. For none of the mixture velocities, the flow has become fully-developed. As suggested by Shah and London (1978), the length required to achieve hydrodynamically fully-developed single-phase flow (L/d 0.055Re), it would take a length of $2.535 \mathrm{~d}$ for the lowest Reynolds number $(\mathrm{Re}=46.1)$ considered in our simulations for the dodecane-water system of fluids. The liquid slug lengths in our cases are between $0.65-0.75 \mathrm{~d}$, hence, a velocity gradient in the radial direction occurs at the beginning of liquid slug, which restricts the flow to show the plug flow behaviour. It can be seen in Figure 12 that at $r / R=0.7$ the liquid near the axis is moving with a higher velocity with respect to the water droplet, while the liquid ahead of $r / R=0.7$ moves with a lower velocity than that of the droplet which indicates the 
International Journal of Mathematical, Engineering and Management Sciences

Vol. 4, No. 6, 1407-1419, 2019

https://dx.doi.org/10.33889/IJMEMS.2019.4.6-111

presence of recirculation inside the liquid slugs where the location of the stagnant liquid is at about $\mathrm{r} / \mathrm{R}=0.7$.

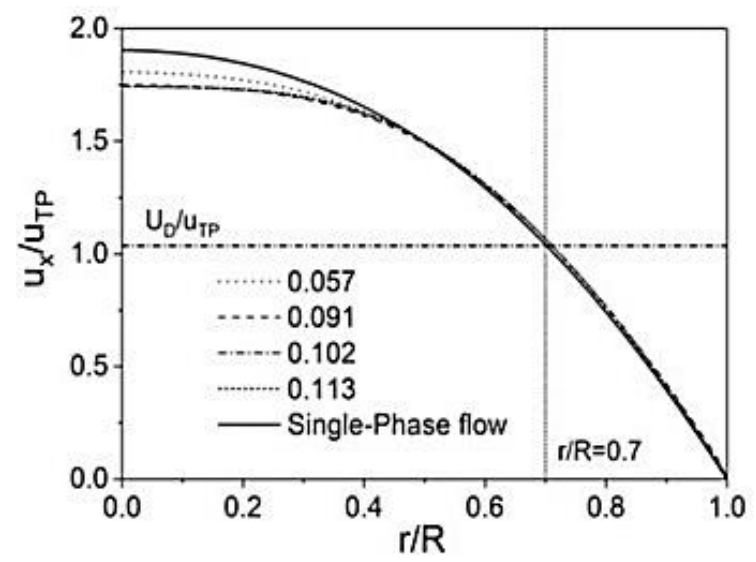

Figure 12. Comparison of the normalized velocity profile in the middle of the liquid slug for four different velocities with the fully-developed single-phase parabolic velocity profile for Do-W system at $\mathrm{Ca}=$ $0.00302, \beta=0.735$, and $\mathrm{L}_{\mathrm{UC}}=6.66 \mathrm{~mm}$. The horizontal dash-dotted line shows the normalized droplet velocity and the vertical dotted line shows the zone of stagnation at $r / R=0.7$

\section{Conclusions}

The numerical study of the flow physics of a two-dimensional, axisymmetric, incompressible, laminar liquid-liquid Taylor flow in a circular channel of diameter $1.5 \mathrm{~mm}$ has been discussed in the present paper. VOF technique was adopted to track the two-phase interface. Two different fluids, namely dodecane and Pd5 were used as the carrier phases, and water was used as the dispersed phase. The effect of Capillary number on various flow parameters was explored. The thickness of the thin liquid film between the wall and droplet was observed to be increasing with mixture velocity. Also, it showed a good match with Bretherton's model (within 4.7\%). The presence of a ripple near the trailing meniscus of the droplet was witnessed, which occurs due to sharp pressure jumps. The pressure gradients in both phases were identical in the region of uniform film thickness. The results of interfacial pressure drop have shown a considerable contribution of approximately 50-55\% and 55-62\% for dodecane-water and Pd5-water systems, respectively, to the overall pressure drop. However, a large deviation in the interfacial pressure drop was observed between the CFD results and Bretherton's model, which suggests the formulation of expression for pressure drop which could cover a wide range of viscosity. The overall pressure drop per unit length of the unit cell was also found to be increasing with the Capillary number. The overall pressure drop in a liquid-liquid flow evaluated from a model available in the literature matches closely with the CFD results for cases having higher viscosity ratios in contrast to that having lower viscosity ratios where the deviations were found to be approximately $40 \%$. Hence, it is strongly recommended to reformulate the existing model of overall pressure drop by considering a large range of viscosity ratios. The present work could be extended to explore the influence of Capillary number on the heat transfer behaviour of liquidliquid Taylor flow in the microcapillary. 
International Journal of Mathematical, Engineering and Management Sciences

Vol. 4, No. 6, 1407-1419, 2019

https://dx.doi.org/10.33889/IJMEMS.2019.4.6-111

\section{Conflict of Interest}

On behalf of all authors, the corresponding author states that there is no conflict of interest.

\section{Acknowledgements}

The authors are grateful to the National Institute of Technology, Warangal (India) for providing the necessary computational facilities to carry out the research work discussed in the present paper.

\section{References}

Asadolahi, A.N., Gupta, R., Fletcher, D.F., \& Haynes, B.S. (2011). CFD approaches for the simulation of hydrodynamics and heat transfer in Taylor flow. Chemical Engineering Science, 66(22), 5575-5584.

Asadolahi, A.N., Gupta, R., Leung, S.S.Y., Fletcher, D.F., \& Haynes, B.S. (2012). Validation of a CFD model of Taylor flow hydrodynamics and heat transfer. Chemical Engineering Science, 69(1), 541552.

Asthana, A., Zinovik, I., Weinmueller, C., \& Poulikakos, D. (2011). Significant Nusselt number increase in microchannels with a segmented flow of two immiscible liquids: An experimental study. International Journal of Heat and Mass Transfer, 54(7-8), 1456-1464.

Aussillous, P., \& Quéré, D. (2000). Quick deposition of a fluid on the wall of a tube. Physics of fluids, 12(10), 2367-2371.

Brackbill, J.U., Kothe, D.B., \& Zemach, C. (1992). A continuum method for modeling surface tension. Journal of Computational Physics, 100(2), 335-354.

Bretherton, F.P. (1961). The motion of long bubbles in tubes. Journal of Fluid Mechanics, 10(2), 166-188.

Farhangi, M.M., Passandideh-Fard, M., \& Moin, H. (2010). Numerical study of bubble rise and interaction in a viscous liquid. International Journal of Computational Fluid Dynamics, 24(1-2), 13-28.

Fouilland, T.S., Fletcher, D.F., \& Haynes, B.S. (2010). Film and slug behaviour in intermittent slug-annular microchannel flows. Chemical Engineering Science, 65(19), 5344-5355.

Gupta, R., Fletcher, D.F., \& Haynes, B.S. (2009). On the CFD modelling of Taylor flow in microchannels. Chemical Engineering Science, 64(12), 2941-2950.

Gupta, R., Leung, S.S., Manica, R., Fletcher, D.F. \& Haynes, B.S. (2013a). Hydrodynamics of liquid-liquid Taylor flow in microchannels. Chemical Engineering Science, 92, 180-189.

Gupta, R., Leung, S.S., Manica, R., Fletcher, D.F. \& Haynes, B.S. (2013b). Three dimensional effects in Taylor flow in circular microchannels. La Houille Blanche, 2013(2), 60-67.

Hirt, C.W. \& Nichols, B.D. (1981). Volume of fluid (VOF) method for the dynamics of free boundaries. Journal of Computational Physics, 39(1), 201-225

Kashid, M.N., Gerlach, I., Goetz, S., Franzke, J., Acker, J.F., Platte, F., Agar, D.W., \& Turek, S. (2005). Internal circulation within the liquid slugs of a liquid-liquid slug-flow capillary microreactor. Industrial and Engineering Chemistry Research, 44(14), 5003-5010.

Lac, E., \& Sherwood, J.D. (2009). Motion of a drop along the centreline of a capillary in a pressure-driven flow. Journal of Fluid Mechanics, 640, 27-54.

Nichita, B.A., Zun, I., \& Thome, J.R. (2010). A level set method coupled with a volume of fluid method for modeling of gas-liquid interface in bubbly flow. Journal of Fluids Engineering, 132(8), 081302. 
International Journal of Mathematical, Engineering and Management Sciences

Vol. 4, No. 6, 1407-1419, 2019

https://dx.doi.org/10.33889/IJMEMS.2019.4.6-111

Ookawara, S., Ishikawa, T., \& Ogawa, K. (2007). Applicability of a miniaturized micro-separator/classifier to oil-water separation. Chemical Engineering and Technology, 30(3), 316-321.

Santos, R., \& Kawaji, M. (2010). Effect of contact angle on gas slug formation, shape and flow in a microchannel T-junction by numerical simulation. In 19th International Congress of Chemical and Process Engineering, 2010, Prague, Czech Republic.

Shah, R.K., \& London, A.L. (1978). Laminar flow forced convection in ducts: a source book for compact heat exchanger analytical data. Academic Press, New York. ISBN: 9780120200511.

Song, H., Chen, D.L., \& Ismagilov, R.F. (2006). Reactions in droplets in microfluidic channels. Angewandte Chemie International Edition, 45(44), 7336-7356.

Taha, T., \& Cui, Z.F. (2006). CFD modelling of slug flow in vertical tubes. Chemical Engineering Science, 61(2), 676-687.

Triplett, K.A., Ghiaasiaan, S.M., Abdel-Khalik, S.I., \& Sadowski, D.L. (1999). Gas-liquid two-phase flow in microchannels Part I: two-phase flow patterns. International Journal of Multiphase Flow, 25(3), 377-394.

Urbant, P., Leshansky, A., \& Halupovich, Y. (2008). On the forced convective heat transport in a dropletladen flow in microchannels. Microfluidics and Nanofluidics, 4(6), 533-542.

Vivekanand, S.V.B., \& Raju, V.R.K. (2015). Simulation of evaporation heat transfer in a rectangular microchannel. Procedia Engineering, 127, 309-316.

White, E.T., \& Beardmore, R.H. (1962). The velocity of rise of single cylindrical air bubbles through liquids contained in vertical tubes. Chemical Engineering Science, 17(5), 351-361. 